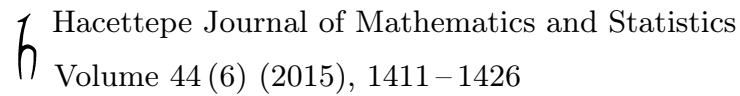

\title{
On the isomorphy of categories of probabilistic limit spaces under t-norms
}

\author{
A.L. Pinchuck* and G. Jäger ${ }^{\dagger \ddagger}$
}

\begin{abstract}
We show that for the classes of strict t-norms, the categories of probabilistic limit spaces under these t-norms are all isomorphic to each other. The same is true for the categories of probabilistic limit spaces under nilpotent t-norms. To show this, we study the isomorphisms between the categories of probabilistic limit spaces under a t-norm, limit tower spaces and approach limit spaces. Similar results are obtained for probabilistic Cauchy spaces and probabilistic uniform limit spaces.
\end{abstract}

Keywords: Probabilistic limit space, probabilistic uniform limit space, probabilistic Cauchy space, limit tower space, uniform limit tower space, Cauchy tower space, convergence approach space, approach Cauchy space, approach uniform limit space, t-norm.

2000 AMS Classification: MSC 54A05, 54A20, 54E15.

Received: 01.04.2013 Accepted: 16.05.2014 Doi: 10.15672/HJMS.2015449654

*Department of Mathematics Rhodes University 6140 Grahamstown, South Africa Email : a.pinchuck@ru.ac.za

${ }^{\dagger}$ School of Mechanical Engineering University of Applied Sciences Stralsund 18435 Stralsund, Germany

Email : gunther.jaeger@fh-stralsund.de

${ }^{\ddagger}$ Corresponding Author. 


\section{Introduction}

Probabilistic limit spaces go back to the work of Florescu [3] and a formulation by means of filter convergence was given by Richardson and Kent [15]. These spaces are extensions of probabilistic metric spaces and probabilistic topological spaces as studied by Menger [12], Schweizer and Sklar [16] and Frank [4]. The category of probabilistic limit spaces is a Cartesian closed, extensional and topological category in the sense of [1]. Triangular norms (t-norms for short) were already used in [16] to model a triangular inequality in probabilistic metric spaces and it therefore seems appropriate to include t-norms in the generalizations of such spaces. This was, consequently, done by Nusser [14] who studied various categories of probabilistic spaces under t-norms.

Ultra-approach limit spaces were introduced by Lowen and Lowen [8] under the name convergence approach spaces. The category of these spaces is a Cartesian closed, extensional and topological category and forms a common framework that encompasses metric spaces and classical convergence spaces. The category of ultra-approach limit

spaces contains the category of approach spaces $[9,10]$ (which form a common framework for topological, metric and uniform spaces) as a reflective subcategory.

In order to study the relationship between probabilistic limit spaces and ultra-approach limit spaces, Brock and Kent [2] introduced the category of limit tower spaces. They could show that the category of probabilistic limit spaces (under the minimum t-norm) is isomorphic to the category of ultra-approach limit spaces.

In this paper, we are extending the results of Brock and Kent [2] to probabilistic limit spaces under a t-norm. In order to do so, we generalize the definition of a limit tower space and introduce a certain subclass of these spaces. It turns out that for certain classes of t-norms, all probabilistic limit spaces under these t-norms are isomorphic. Similar results can be shown for probabilistic Cauchy spaces under a t-norm and for probabilistic uniform limit spaces under a t-norm.

We are finally going to introduce the basic concepts that we need later and fix the notation. A $t$-norm $*:[0,1] \times[0,1] \longrightarrow[0,1]$ is a binary operation on $[0,1]$ which is associative, commutative, non-decreasing in each argument and which has 1 as the unit. A t-norm is called continuous if it is continuous as a mapping from $[0,1] \times[0,1] \longrightarrow$ $[0,1]$. A special class of t-norms is given by continuous Archimedean t-norms. These are determined by continuous, strictly decreasing additive generators $S:[0,1] \longrightarrow[0, \infty]$ with $S(1)=0$ such that

$$
\alpha * \beta=S^{(-1)}(S(\alpha)+S(\beta))
$$


with the pseudo-inverse $S^{(-1)}(u)=\bigvee\{x \in[0,1] \quad: S(x)>u\}=\left\{\begin{array}{ll}v & \text { if } S(v)=u \\ 0 & \text { if } u>S(0)\end{array}\right.$. Note that $\bigvee \emptyset=\bigwedge[0,1]=0$ here.

We further note that the pseudo-inverse $S^{(-1)}:[0, \infty] \longrightarrow[0,1]$ is continuous, surjective and strictly decreasing on $[0, S(0)]$ and that $S\left(S^{(-1)}(u)\right)=u$ if $u \leq S(0)$ and that $S^{(-1)}(S(u))=u$ for all $u \in[0,1]$. Continuous Archimedean t-norms can be separated into two classes.

- $S(0)=\infty$. These are the strict $t$-norms. In this case $S^{(-1)}=S^{-1}$. A typical example is the product t-norm $\alpha * \beta=\alpha \beta$ with additive generator $S(x)=-\ln (x)$ (and $S(0)=\infty$ ).

- $S(0)<\infty$. These are the nilpotent $t$-norms. Noting that for an additive generator $S$ for a continuous Archimedean t-norm and for all $a>0, \bar{S}(x)=a S(x)$ defines an additive generator for the same t-norm, we can always assume for a nilpotent t-norm that $S(0)=1$. A typical example for a nilpotent $\mathrm{t}$-norm is the Lukasiewicz t-norm $\alpha * \beta=(\alpha+\beta-1) \vee 0$ with additive generator $S(x)=1-x$.

An example of a non-Archimediean t-norm is the minimum t-norm $\alpha * \beta=\alpha \wedge \beta$. For further results on t-norms we refer to Schweizer and Sklar [16] and to [6].

We finally fix some notation. For a set $X$, we denote $P(X)$ its power set. We denote the set of all filters $\mathbb{F}, \mathbb{G}, \mathbb{H}, \ldots$ on the set $X$ by $\mathbb{F}(X)$. We order this set by set inclusion and we denote for $x \in X$ the point filter by $[x]=\{F \subseteq X: x \in F\}$. For a subset $A$ of an ordered set $X$ we write, in case of existence, $\bigvee A$ for its supremum and $\bigwedge A$ for its infimum. If $A=\{\alpha, \beta\}$, then we write $\alpha \wedge \beta=\wedge A$ and $\alpha \vee \beta=\bigvee A$. For notions from category theory we refer to [1].

\section{Probabilistic limit spaces, limit tower spaces and approach con- vergence spaces}

A probabilistic limit space under a t-norm * [14] is a pair $(X, \bar{q})$ of a set $X$ and a nonempty family of mappings $\bar{q}=\left(q_{\lambda}: \mathbb{F}(X) \longrightarrow P(X)\right)_{\lambda \in[0,1]}$ that satisfies the following axioms.

(PL1) $x \in q_{\alpha}([x])$ for all $\alpha \in[0,1], x \in X$;

(PL2) $q_{\alpha}(\mathbb{F}) \subseteq q_{\alpha}(\mathbb{G})$, whenever $\mathbb{F} \leq \mathbb{G}$;

(PL3) $q_{\beta}(\mathbb{F}) \subseteq q_{\alpha}(\mathbb{F})$ whenever $\alpha \leq \beta$;

(PL4) $q_{0}(\mathbb{F})=X$

(PL5) $x \in q_{\alpha * \beta}(\mathbb{F} \wedge \mathbb{G})$ whenever $x \in q_{\alpha}(\mathbb{F})$ and $x \in q_{\beta}(\mathbb{G})$;

$(\mathrm{PLLC}) \quad q_{\alpha}(\mathbb{F})=\bigcap_{\beta<\alpha} q_{\beta}(\mathbb{F}) ;$ for all $\alpha, \beta \in[0,1], \mathbb{F}, \mathbb{G} \in \mathbb{F}(X)$. 
The condition (PLLC) is called left-continuity. It is not required in the original definition by Nusser [14], however we will need it later. A mapping $f: X \longrightarrow X^{\prime}$ between the probabilistic limit spaces under the t-norm $*,(X, \bar{q}),\left(X^{\prime}, \bar{q}^{\prime}\right)$, is continuous if for all $\alpha \in[0,1]$ and all $\mathbb{F} \in \mathbb{F}(X)$ we have $f\left(q_{\alpha}(\mathbb{F})\right) \subseteq q_{\alpha}^{\prime}(f(\mathbb{F}))$. The category of all probabilistic limit spaces under the t-norm $*$ with the continuous mappings as morphisms is denoted by $P L I M^{*}$. It is shown in [14] that $P L I M^{*}$ is a topological and extensional construct and for $*=\wedge, P L I M^{\wedge}$ is Cartesian closed.

2.1. Lemma. Let $(X, \bar{q})$ be a probabilistic limit space under the minimum t-norm $\wedge$. Then (PL5) is equivalent to the axiom

$(u P L 5) \quad x \in q_{\alpha}(\mathbb{F} \wedge \mathbb{G})$ whenever $x \in q_{\alpha}(\mathbb{F})$ and $x \in q_{\alpha}(\mathbb{G})$.

Proof. If (PL5) is true, then we simply choose $\alpha=\beta$. If (uPL5) is true, then for $x \in q_{\alpha}(\mathbb{F}) \cap q_{\beta}(\mathbb{G})$ we have, because $\alpha \wedge \beta \leq \alpha, \beta$ and (PL3) that $x \in q_{\alpha \wedge \beta}(\mathbb{F}) \cap q_{\alpha \wedge \beta}(\mathbb{G})$ and hence, by (uPL5), also $x \in q_{\alpha \wedge \beta}(\mathbb{F} \wedge \mathbb{G})$.

Therefore, probabilistic limit spaces under the minimum t-norm $\wedge$ are (left-continuous) componentwise probabilistic limit spaces in the definition of [14].

A limit tower space is a pair $(X, \bar{p})$ of a set $X$ and a non-empty family of mappings $\bar{p}=\left(p_{\epsilon}: \mathbb{F}(X) \longrightarrow P(X)\right)_{\epsilon \in[0, \infty]}$ that satisfies the following axioms.

(LT1) $x \in p_{\epsilon}([x])$ for all $\epsilon \in[0, \infty], x \in X$;

(LT2) $p_{\epsilon}(\mathbb{F}) \subseteq p_{\epsilon}(\mathbb{G})$, whenever $\mathbb{F} \leq \mathbb{G} ;$

(LT3) $p_{\delta}(\mathbb{F}) \subseteq p_{\epsilon}(\mathbb{F})$ whenever $\delta \leq \epsilon$;

(LT4) $p_{\infty}(\mathbb{F})=X$;

(LT5) $x \in p_{\epsilon+\delta}(\mathbb{F} \wedge \mathbb{G})$ whenever $x \in p_{\epsilon}(\mathbb{F})$ and $x \in p_{\delta}(\mathbb{G})$;

(LTLC) $\quad p_{\epsilon}(\mathbb{F})=\bigcap_{\epsilon<\delta} p_{\delta}(\mathbb{F})$, for all $\epsilon, \delta \in[0, \infty], \mathbb{F}, \mathbb{G} \in \mathbb{F}(X)$.

The condition (LTLC) is again called left-continuity. A mapping $f: X \longrightarrow X^{\prime}$ between the limit tower spaces $(X, \bar{p}),\left(X^{\prime}, \bar{p}^{\prime}\right)$ is continuous if for all $\epsilon \in[0, \infty]$ and all $\mathbb{F} \in \mathbb{F}(X)$ we have $f\left(p_{\epsilon}(\mathbb{F})\right) \subseteq p_{\epsilon}^{\prime}(f(\mathbb{F}))$. The category of all limit tower spaces with the continuous mappings as morphisms is denoted by LTS.

If we replace the axiom (LT5) by the axiom $\left(\right.$ uLT5) $\quad x \in p_{\epsilon \vee \delta}(\mathbb{F} \wedge \mathbb{G})$ whenever $x \in p_{\epsilon}(\mathbb{F})$ and $x \in p_{\delta}(\mathbb{G})$; then we speak of a ultra-limit tower space. The category of ultra-limit tower spaces with continuous mappings as morphisms is denoted by $u L T S$.

2.2. Lemma. Let $(X, \bar{p})$ be an ultra-limit tower space. Then (uLT5) is equivalent to the axiom

$\left(u L T 5^{\prime}\right) \quad x \in p_{\epsilon}(\mathbb{F} \wedge \mathbb{G})$ whenever $x \in p_{\epsilon}(\mathbb{F})$ and $x \in p_{\epsilon}(\mathbb{G})$.

Proof. Similar to the proof of Lemma 2.1. 
The preceding Lemma shows that ultra-limit tower spaces are the same as limit tower spaces as originally introduced and studied in [2]. We prefer to rename them in the light of the subsequent sections.

An approach limit space [11] is a pair $(X, \lambda)$ of a set $X$ and a mapping $\lambda: \mathbb{F}(X) \longrightarrow$ $[0, \infty]^{X}$ that satisfies the following axioms.

(AL1) $\lambda([x])(x)=0$ for all $x \in X$;

$(\mathrm{AL} 2) \quad \lambda(\mathbb{G})(x) \leq \lambda(\mathbb{F})(x)$ whenever $\mathbb{F} \leq \mathbb{G}$;

(AL3) $\quad \lambda(\mathbb{F} \wedge \mathbb{G})(x) \leq \lambda(\mathbb{F})(x)+\lambda(\mathbb{G})(x)$.

The value $\lambda(\mathbb{F})(x)$ has the interpretation as the distance that $x$ is away from being a limit point of $\mathbb{F}[10]$. A mapping $f: X \longrightarrow X^{\prime}$ between two approach limit spaces $(X, \lambda)$, $\left(X^{\prime}, \lambda^{\prime}\right)$ is called a contraction if for all $\mathbb{F} \in \mathbb{F}(X)$ and all $x \in X$ we have $\lambda^{\prime}(f(\mathbb{F}))(f(x)) \leq$ $\lambda(\mathbb{F})(x)$

If we replace the axiom (AC3) by the stronger axiom $($ uAL3) $\quad \lambda(\mathbb{F} \wedge \mathbb{G})(x) \leq \lambda(\mathbb{F})(x) \vee \lambda(\mathbb{G})(x)$

then we call the pair $(X, \lambda)$ an ultra-approach limit space. Note that these spaces were originally called convergence approach spaces and introduced and studied by Lowen and Lowen [8]. What we call here an approach limit space is called weak convergence approach space in [11]. Again we prefer to change the names in order to reach consistency with other notations. The category of approach limit spaces with contractions as morphisms is denoted by $A L S$, the subcategory of ultra-approach limit spaces is denoted by $u A L S$. The category $u A L S$ is topological, extensional and Cartesian closed [8] and whereas $A L S$ is topological and contains $u A L S$ as a bireflective subcategory [11].

\section{Isomorphisms between the categories $A L S$ and $L T S$}

The following isomorphism functors between the categories of ultra-approach limit spaces and ultra-limit tower spaces were introduced in [2]. We extend their definition to the categories of approach limit spaces and limit tower spaces.

For $(X, \lambda) \in|u A L S|$ we define $\eta \lambda=\left((\eta \lambda)_{\epsilon}\right)_{\epsilon \in[0, \infty]}$ by

$$
x \in(\eta \lambda)_{\epsilon}(\mathbb{F}) \quad \Longleftrightarrow \quad \lambda(\mathbb{F})(x) \leq \epsilon .
$$

It is shown in [2] that $\eta: u A L S \longrightarrow u L T S,(X, \lambda) \longmapsto(X, \eta \lambda), f \longmapsto f$ is a functor.

For $(X, \bar{p}) \in|u L T S|$ we define $\rho \bar{p}: \mathbb{F}(X) \longrightarrow[0, \infty]^{X}$ by

$$
(\rho \bar{p})(\mathbf{F})(x)=\bigwedge\left\{\epsilon \in[0, \infty]: x \in p_{\epsilon}(\mathbb{F})\right\} .
$$

It is shown in [2] that $\rho: u L T S \longrightarrow u A L S,(X, \bar{p}) \longmapsto(X, \rho \bar{p}), f \longmapsto f$ is a functor and that $\eta \circ \rho=i d_{u L T S}$ and $\rho \circ \eta=i d_{u A C P}$. Hence both functors are isomorphism functors and the categories $u A L S$ and $u L T S$ are isomorphic. We will show with the next two 
lemmas that both functors can be extended to the categories $A L S$ and $L T S$. To this end, we simply use for $(X, \lambda) \in|A L S|$, resp. for $(X, \bar{p}) \in|L T S|$ the same definitions of $\eta \lambda$ and $\rho \bar{p}$, i.e. we extend the domains of the functors $\eta$ and $\rho$ to $A L S$ and $L T S$, respectively. We will show that the co-domains then are again the categories $L T S$ and $A L S$, respectively.

3.1. Lemma. Let $(X, \lambda) \in|A L S|$. Then $(X, \eta \lambda)$ satisfies the axiom (LT5).

Proof. Let $x \in(\eta \lambda)_{\epsilon}(\mathbb{F})$ and $x \in(\eta \lambda)_{\delta}(\mathbb{G})$. Then $\lambda(\mathbb{F})(x) \leq \epsilon$ and $\lambda(\mathbb{G})(x) \leq \delta$. By $\left(\right.$ AC3) then $\lambda(\mathbb{F} \wedge \mathbb{G})(x) \leq \epsilon+\delta$, i.e. $x \in(\eta \lambda)_{\epsilon+\delta}(\mathbb{F} \wedge \mathbb{G})$.

3.2. Lemma. Let $(X, \bar{p}) \in|L T S|$. Then $(X, \rho \bar{p})$ satisfies the axiom (AC3).

Proof. Let $\rho \bar{p}(\mathbb{F})(x)=\epsilon$ and $\rho \bar{p}(\mathbb{G})(x)=\delta$. For $\epsilon^{\prime}>\epsilon$ and $\delta^{\prime}>\delta$ then $x \in p_{\epsilon^{\prime}}(\mathbb{F})$ and $x \in p_{\delta^{\prime}}(\mathbb{G})$. By $\left(\right.$ LT5) then $x \in p_{\epsilon^{\prime}+\delta^{\prime}}(\mathbb{F} \wedge \mathbb{G})$ and hence $\rho \bar{p}(\mathbb{F} \wedge \mathbb{G})(x) \leq \epsilon^{\prime}+\delta^{\prime}$. As $\epsilon^{\prime}>\epsilon$ and $\delta^{\prime}>\delta$ are arbitrary we conclude $\rho \bar{p}(\mathbb{F} \wedge \mathbb{G})(x) \leq \epsilon+\delta=\rho \bar{p}(\mathbb{F})(x)+\rho \bar{p}(\mathbb{G})(x)$.

3.3. Theorem. The categories ALS and LTS are isomorphic.

\section{The case of strict t-norms}

Let now $*$ be a strict t-norm with additive generator $S:[0,1] \longrightarrow[0, \infty]$. Brock and Kent [2] have defined the following isomorphism functors between the categories $P L I M^{\wedge}$ and $u L T S$. For an ultra-limit tower space $(X, \bar{p})$ we define $\left(\Phi_{S} \bar{p}\right)_{\alpha}=p_{S(\alpha)}$. Then $\Phi_{S}: u L T S \longrightarrow P L I M^{\wedge},(X, \bar{p}) \longmapsto\left(X, \Phi_{S} \bar{p}\right), f \longmapsto f$ is a functor. For a levelwise probabilistic limit space $(X, \bar{q})$ we define $\left(\Psi_{S} \bar{q}\right)_{\epsilon}=q_{S^{-1}(\epsilon)}$. Then $\Psi_{S}: P L I M^{\wedge} \longrightarrow$ $u \operatorname{LTS},(X, \bar{q}) \longmapsto\left(X, \Psi_{S} \bar{q}\right), f \longmapsto f$ is a functor and $\Phi_{S} \circ \Psi_{S}=i d_{P L I M} \wedge$ and $\Psi_{S} \circ \Phi_{S}=$ $i d_{u L T S}$. Hence both functors are isomorphism functors and $P L I M^{\wedge}$ and $u L T S$ are isomorphic. We will show with the next two lemmas that these functors can be extended to the categories $P L I M^{*}$ and $L T S$, provided that $*$ is the strict t-norm generated by $S$.

4.1. Lemma. Let the strict $t$-norm * have the additive generator $S$ and let $(X, \bar{q}) \in$ $\left|P L I M^{*}\right|$. Then $\left(X, \Psi_{S} \bar{q}\right)$ satisfies the axiom (LT5).

Proof. Let $x \in\left(\Psi_{S} \bar{q}\right)_{\epsilon}(\mathbb{F})$ and $x \in\left(\Psi_{S} \bar{q}\right)_{\delta}(\mathbb{G})$. Then $x \in q_{S^{-1}(\epsilon)}(\mathbb{F})$ and $x \in q_{S^{-1}(\delta)}(\mathbb{G})$ and by (PL5) then $x \in q_{S^{-1}(\epsilon) * S^{-1}(\delta)}(\mathbb{F} \wedge \mathbb{G})$. By the definition of the t-norm $*$ it is easily verified that $S^{-1}(\epsilon) * S^{-1}(\delta)=S^{-1}(\epsilon+\delta)$ and hence $x \in q_{S^{-1}(\epsilon+\delta)}(\mathbb{F} \wedge \mathbb{G})$, which means $x \in\left(\Psi_{S} \bar{q}\right)_{\epsilon+\delta}(\mathbb{F} \wedge \mathbb{G})$.

4.2. Lemma. Let the strict $t$-norm * have the additive generator $S$ and let $(X, \bar{p}) \in$ $|L T S|$. Then $\left(X, \Phi_{S} \bar{p}\right)$ satisfies the axiom (PL5). 
Proof. Let $x \in\left(\Phi_{S} \bar{p}\right)_{\alpha}(\mathbb{F})$ and $x \in\left(\Phi_{S} \bar{p}\right)_{\beta}(\mathbb{G})$. Then $x \in p_{S(\alpha)}(\mathbb{F})$ and $x \in p_{S(\beta)}(\mathbb{G})$ and hence by $\left(\right.$ LT5) $x \in p_{S(\alpha)+S(\beta)}(\mathbb{F} \wedge \mathbb{G})$. By definition of the t-norm $*$ we see that $S(\alpha)+S(\beta)=S(\alpha * \beta)$ and hence $x \in p_{S(\alpha * \beta)}(\mathbb{F} \wedge \mathbb{G})$. But this means that $x \in$ $\left(\Phi_{S} \bar{p}\right)_{\alpha * \beta}(\mathbb{F} \wedge \mathbb{G})$.

4.3. Corollary. For a strict t-norm *, the categories PLIM* and LTS are isomorphic.

We conclude the following main result of this section.

4.4. Theorem. For strict $t$-norms, all categories PLIM* are isomorphic.

\section{The case of nilpotent t-norms}

We are now showing similar results for the class of nilpotent t-norms. To this end, we first introduce a subcategory of $L T S$.

For $\omega \in(0, \infty]$ we call $(X, \bar{p}) \in|L T S|$ an $\omega$-limit tower space if the following strengthening of (LT4) is valid:

$($ LT4 $\omega) \quad p_{\epsilon}(\mathbb{F})=X$ whenever $\omega \leq \epsilon$.

We see that a limit tower space is the same as an $\infty$-limit tower space. The subcategory of $L T S$ with objects the $\omega$-limit tower spaces is denoted by $L T S_{\omega}$. It is not difficult to show that $L T S_{\omega}$ is a bireflective subcategory of $L T S$.

We consider now a nilpotent t-norm with additive generator $S$. We will show that $P L I M^{*}$ and $L T S_{S(0)}$ are isomorphic. To this end, we generalize the two functors of the previous section. For $(X, \bar{q}) \in\left|P L I M^{*}\right|$ we define $(\Psi \bar{q})_{\epsilon}=q_{S^{(-1)}(\epsilon)}$.

5.1. Lemma. For $(X, \bar{q}) \in\left|P L I M^{*}\right|$ we have that $(X, \Psi \bar{q}) \in\left|L T S_{S(0)}\right|$.

Proof. (LT1) and (LT2) are easy. For (LT3) we may assume $\epsilon \leq \delta<S(0)$. Then $S^{(-1)}(\epsilon) \geq S^{(-1)}(\delta)$ and hence $(\Psi \bar{q})_{\epsilon}=q_{S^{(-1)}(\epsilon)} \subseteq q_{S^{(-1)}(\delta)}=(\Psi \bar{q})_{\delta}$.

For $\left(\operatorname{LT}_{S(0)}\right)$, let $\epsilon \geq S(0)$. Then $S^{(-1)}(\epsilon)=0$ and hence $(\Psi \bar{q})_{\epsilon}(\mathbb{F})=q_{0}(\mathbb{F})=X$.

For (LT5), let $x \in(\Psi \bar{q})_{\epsilon}(\mathbb{F}) \cap(\Psi \bar{q})_{\delta}(\mathbb{G})$. If $\epsilon+\delta \geq S(0)$, then there is nothing to prove. If $\epsilon+\delta<S(0)$, then both $\epsilon, \delta<0$ and hence $S^{(-1)}(\epsilon) * S^{(-1)}(\delta)=S^{(-1)}(\epsilon+\delta)$ and we conclude

$$
\begin{aligned}
& (\Psi \bar{q})_{\epsilon}(\mathbb{F}) \cap(\Psi \bar{q})_{\delta}(\mathbb{G})=q_{S^{(-1)}(\epsilon)}(\mathbb{F}) \cap q_{S^{(-1)}(\delta)}(\mathbb{G}) \subseteq q_{S^{(-1)}(\epsilon) * S^{(-1)}(\delta)}(\mathbb{F} \wedge \mathbb{G}) \\
& =q_{S^{(-1)}(\epsilon+\delta)}(\mathbb{F} \wedge \mathbb{G})=(\Psi \bar{q})_{\epsilon+\delta}(\mathbb{F} \wedge \mathbb{G}) .
\end{aligned}
$$

We finally show (LTLC). If $\epsilon \geq S(0)$ then for $\delta>\epsilon$ we have $(\Psi \bar{q})_{\delta}(\mathbb{F})=X$ and hence $(\Psi \bar{q})_{\epsilon}(\mathbb{F})=X=\bigcap_{\delta>\epsilon}(\Psi \bar{q})_{\delta}(\mathbb{F})$. If $\epsilon<S(0)$ then by continuity and surjectivity of $S^{(-1)}$ 
and because $S^{(-1)}$ is strictly decreasing on $[0, S(0)]$, for $\beta<S^{(-1)}(\epsilon)$ there is a unique $\delta \in(\epsilon, S(0)]$ such that $\beta=S^{(-1)}(\delta)$. Hence

$$
(\Psi \bar{q})_{\epsilon}(\mathbb{F})=q_{S^{(-1)}(\epsilon)}(\mathbb{F})=\bigcap_{\beta<S^{(-1)}(\epsilon)} q_{\beta}(\mathbb{F})=\bigcap_{S^{(-1)}(\delta)<S^{(-1)}(\epsilon)} q_{S^{(-1)}(\delta)}(\mathbb{F}) .
$$

Now for $\epsilon<\delta \leq S(0), S^{(-1)}(\delta)<S^{(-1)}(\epsilon)$ is equivalent to $\epsilon<\delta$ and hence we obtain

$$
(\Psi \bar{q})_{\epsilon}(\mathbb{F})=\bigcap_{\epsilon<\delta} q_{S}(-1)(\delta)(\mathbb{F})=\bigcap_{\epsilon<\delta}(\Psi \bar{q})_{\delta}(\mathbb{F}) .
$$

It follows easily from this that $\Psi: P L I M^{*} \longrightarrow \operatorname{LTS}_{S(0)},(X, \bar{q}) \longmapsto(X, \Psi \bar{q}), f \longmapsto f$ is a functor.

For $(X, \bar{p}) \in\left|L T S_{S(0)}\right|$ we define now $(\Phi \bar{p})_{\alpha}=p_{S(\alpha)}$.

5.2. Lemma. For $(X, \bar{p}) \in\left|L T S_{S(0)}\right|$ we have that $(X, \Phi \bar{p}) \in\left|P L I M^{*}\right|$.

Proof. (PL1) and (PL2) are again easy. (PL3) follows because $S$ is order-reversing. For $(\mathrm{PL} 4)$ we note that $(\Phi \bar{p})_{0}(\mathbb{F})=p_{S(0)}(\mathbb{F})=X$. For $(\mathrm{PL} 5)$, we have

$$
(\Phi \bar{p})_{\alpha}(\mathbb{F}) \cap(\Phi \bar{p})_{\beta}(\mathbb{G})=p_{S(\alpha)}(\mathbb{F}) \cap p_{S(\beta)}(\mathbb{G}) \subseteq P_{S(\alpha)+S(\beta)}(\mathbb{F} \wedge \mathbb{G}) .
$$

By definition of the t-norm we have $S(\alpha * \beta)=S\left(S^{(-1)}(S(\alpha)+S(\beta))\right)$. We distinguish two cases. If $S(\alpha)+S(\beta) \leq S(0)$, then $S(\alpha * \beta)=S(\alpha)+S(\beta)$. Then

$$
(\Phi \bar{p})_{\alpha}(\mathbb{F}) \cap(\Phi \bar{p})_{\beta}(\mathbb{G}) \subseteq p_{S(\alpha * \beta)}(\mathbb{F} \wedge \mathbb{G})=(\Phi \bar{p})_{\alpha * \beta}(\mathbb{F} \wedge \mathbb{G}) .
$$

If $S(\alpha)+S(\beta)>S(0)$, then $S(\alpha * \beta)=S(0)$ and hence

$$
\begin{aligned}
& (\Phi \bar{p})_{\alpha}(\mathbb{F}) \cap(\Phi \bar{p})_{\beta}(\mathbb{G}) \subseteq p_{S(\alpha)+S(\beta)}(\mathbb{F} \wedge \mathbb{G})=X \\
& =p_{S(0)}(\mathbb{F} \wedge \mathbb{G})=p_{S(\alpha * \beta)}(\mathbb{F} \wedge \mathbb{G})=(\Phi \bar{p})_{\alpha * \beta}(\mathbb{F} \wedge \mathbb{G}) .
\end{aligned}
$$

The axiom (PLLC) finally follows with similar arguments as the proof of (LTLC) in the previous Lemma.

It follows easily from this that $\Phi: L T S_{S(0)} \longrightarrow P L I M^{*},(X, \bar{p}) \longmapsto(X, \Phi \bar{p}), f \longmapsto f$ is a functor. Now we note that $(\Phi \circ \Psi \bar{q})_{\alpha}=q_{S^{(-1)}(S(\alpha))}=q_{\alpha}$. If $\epsilon \leq S(0)$, then $S\left(S^{(-1)}(\epsilon)\right)=\epsilon$ and hence $(\Psi \circ \Phi \bar{p})_{\epsilon}=p_{S\left(S^{(-1)}(\epsilon)\right)}=p_{\epsilon}$. If $\epsilon>S(0)$ then trivially $(\Psi \circ \bar{p})_{\epsilon}=X=p_{\epsilon}$. Hence both functors, $\Psi$ and $\Phi$ are isomorphism functors and we can state the following result.

5.3. Lemma. PLIM* and $L T S_{S(0)}$ are isomorphic categories.

As noted above, for a nilpotent t-norm, we can always assume that $S(0)=1$ for an additive generator. Hence we obtain the following result. 
5.4. Theorem. For nilpotent t-norms, all categories PLIM* are isomorphic.

\section{Probabilistic Cauchy spaces, Cauchy tower spaces and approach Cauchy spaces}

A probabilistic Cauchy space under the t-norm $*[14]$ is a pair $(X, \bar{C})$ of a set $X$ and a non-empty family of subsets of $\mathbb{F}(X), \bar{C}=\left(C_{\alpha}\right)_{\alpha \in[0,1]}$, that satisfies the following axioms.

(PC1) $[x] \in C_{\alpha}$ for all $x \in X$ and all $\alpha \in[0,1]$;

(PC2) $\mathbb{G} \in C_{\alpha}$ whenever $\mathbb{F} \in C_{\alpha}$ and $\mathbb{F} \leq \mathbb{G}$;

(PC3) $C_{\beta} \subseteq C_{\alpha}$ whenever $\alpha \leq \beta$;

(PC4) $C_{0}=\mathbb{F}(X)$;

(PC5) $\mathbb{F} \wedge \mathbb{G} \in C_{\alpha * \beta}$ whenever $\mathbb{F} \in C_{\alpha}, \mathbb{G} \in C_{\beta}$ and $\mathbb{F} \vee \mathbb{G}$ exists;

(PCLC) $\quad C_{\alpha}=\bigcap_{\beta<\alpha} C_{\beta}$.

A mapping $f: X \longrightarrow X^{\prime}$ between two probabilistic Cauchy spaces under the t-norm $*,(X, \bar{C}),\left(X, \bar{C}^{\prime}\right)$, is called Cauchy-continuous if for all $\alpha \in[0,1]$ we have $f\left(C_{\alpha}\right) \subseteq C_{\alpha}^{\prime}$. The category of probabilistic Cauchy spaces under the t-norm $*$ and Cauchy continuous mappings is denoted by $P C h y^{*}$.

6.1. Lemma. Let $(X, \bar{C})$ be a probabilistic Cauchy space under the t-norm $\wedge$. Then (PC5) is equivalent to the axiom

$(u P C 5) \quad \mathbb{F} \wedge \mathbb{G} \in C_{\alpha}$ whenever $\mathbb{F} \in C_{\alpha}$ and $\mathbb{G} \in C_{\alpha}$ and $\mathbb{F} \vee \mathbb{G}$ exists.

Proof. If (PC5) is true, then we simply choose $\alpha=\beta$. If (uPC5) is true, then for $\mathbb{F} \in C_{\alpha}$ and $\mathbb{G} \in C_{\beta}$ we conclude with (PC3) that $\mathbb{F} \in C_{\alpha \wedge \beta}$ and $\mathbb{G} \in C_{\alpha \wedge \beta}$. Therefore, if $\mathbb{F} \vee \mathbb{G}$ exists, by (uPC5) then $\mathbb{F} \wedge \mathbb{G} \in C_{\alpha \wedge \beta}$.

Therefore, probabilistic Cauchy spaces under the t-norm $\wedge$ are (left-continuous) componentwise probabilistic Cauchy spaces in the definition of [14]. The category PChy* is topological but it is not hereditary and quotients are not productive, not even for $*=\wedge$. However, $P C h y^{\wedge}$ is Cartesian closed, see [14].

A Cauchy tower space is a pair $(X, \bar{D})$ of a set $X$ and a non-empty family of subsets of $\mathbb{F}(X), \bar{D}=\left(D_{\epsilon}\right)_{\epsilon \in[0, \infty]}$, that satisfies the following axioms.

(CT1) $[x] \in D_{\epsilon}$ for all $x \in X$ and all $\epsilon \in[0, \infty]$;

$(\mathrm{CT} 2) \quad \mathbb{G} \in D_{\epsilon}$ whenever $\mathbb{F} \in D_{\epsilon}$ and $\mathbb{F} \leq \mathbb{G}$;

(CT3) $D_{\epsilon} \subseteq D_{\delta}$ whenever $\epsilon \leq \delta$;

(CT4) $D_{\infty}=\mathbb{F}(X)$;

(CT5) $\mathbb{F} \wedge \mathbb{G} \in D_{\epsilon+\delta}$ whenever $\mathbb{F} \in D_{\epsilon}, \mathbb{G} \in D_{\delta}$ and $\mathbb{F} \vee \mathbb{G}$ exists;

(CTLC) $D_{\epsilon}=\bigcap_{\epsilon<\delta} C_{\delta}$. 
A mapping $f: X \longrightarrow X^{\prime}$ between two Cauchy tower spaces, $(X, \bar{D}),\left(X, \bar{D}^{\prime}\right)$, is called Cauchy-continuous if for all $\epsilon \in[0, \infty]$ we have $f\left(D_{\epsilon}\right) \subseteq D_{\epsilon}^{\prime}$. The category of Cauchy tower spaces and Cauchy continuous mappings is denoted by ChyTS.

If we replace the axiom (CT5) by the axiom (uCT5) $\mathbb{F} \wedge \mathbb{G} \in D_{\epsilon \vee \delta}$ whenever $\mathbb{F} \in D_{\epsilon}, \mathbb{G} \in D_{\delta}$ and $\mathbb{F} \vee \mathbb{G}$ exists; then we speak of a ultra-Cauchy tower space. The category of ultra-Cauchy tower spaces with continuous mappings as morphisms is denoted by $u C h y T S$.

6.2. Lemma. Let $(X, \bar{D})$ be an ultra-Cauchy tower space. Then (uCT5) is equivalent to the axiom

$\left(u C T 5^{\prime}\right) \quad \mathbb{F} \wedge \mathbb{G} \in D_{\epsilon}$ whenever $\mathbb{F} \in D_{\epsilon}$ and $\mathbb{G} \in D_{\epsilon}$ and $\mathbb{F} \vee \mathbb{G}$ exists.

Proof. Similar to the proof of Lemma 6.1.

We note that Cauchy tower spaces are defined in a different way in [13]. We define for $0<\omega \leq \infty$ an $\omega$-Cauchy tower space as a Cauchy tower space that satisfies the following strengthening of (CT4)

$(\mathrm{CT} 4 \omega) \quad D_{\epsilon}=\mathbb{F}(X)$ whenever $\omega \leq \epsilon$.

We denote the subcategory of $C T S$ with objects the $\omega$-Cauchy tower spaces by $C T S_{\omega}$. It is not difficult to prove that $C T S_{\omega}$ is bireflective in $C T S$.

An approach Cauchy space [11] is a pair $(X, \gamma)$ of a set $X$ and a mapping $\gamma: \mathbb{F}(X) \longrightarrow$ $[0, \infty]$ that satisfies the following axioms.

(AChy1) $\gamma([x])=0$ for all $x \in X$;

(AChy2) $\gamma(\mathbb{G}) \leq \gamma(\mathbb{F})$ whenever $\mathbb{F} \leq \mathbb{G}$;

(AChy3) $\quad \gamma(\mathbb{F} \wedge \mathbb{G}) \leq \gamma(\mathbb{F})+\gamma(\mathbb{G})$ whenever $\mathbb{F} \vee \mathbb{G}$ exists.

A mapping $f: X \longrightarrow X^{\prime}$ between two approach Cauchy spaces $(X, \gamma),\left(X^{\prime}, \gamma^{\prime}\right)$ is called a Cauchy contraction if for all $\mathbb{F} \in \mathbb{F}(X)$ we have $\gamma^{\prime}(f(\mathbb{F})) \leq \gamma(\mathbb{F})$

If we replace the axiom (AChy3) by the stronger axiom (uAChy3) $\quad \gamma(\mathbb{F} \wedge \mathbb{G}) \leq \gamma(\mathbb{F}) \vee \gamma(\mathbb{G})$ whenever $\mathbb{F} \vee \mathbb{G}$ exists; then we call the pair $(X, \gamma)$ an ultra-approach Cauchy space.

The category of approach Cauchy spaces with Cauchy contractions as morphisms is denoted by AChy, the subcategory of ultra-approach convergence spaces is denoted by $u A C h y$. The category $u A C h y$ is a bireflective subcategory of AChy. AChy is topological and $u A C h y$ is also Cartesian closed [11].

We can define isomorphism functors between the categories in a similar way as in the previous section. For $(X, \gamma) \in|A C h y|$ we define the Cauchy tower $\sigma \gamma$ by

$$
\mathbb{F} \in(\sigma \gamma)_{\epsilon} \Longleftrightarrow \gamma(\mathbb{F}) \leq \epsilon
$$


For $(X, \bar{D}) \in|C T S|$ we define the mapping $\tau \bar{D}: \mathbb{F}(X) \longrightarrow[0, \infty]$ by

$$
\tau \bar{D}(\mathbb{F})=\bigwedge\left\{\epsilon \in[0, \infty]: \mathbb{F} \in D_{\epsilon}\right\}
$$

The following result is not difficult to prove.

6.3. Lemma. (1) $\sigma: A C h y \longrightarrow C T S,(X, \gamma) \longmapsto(X, \sigma \gamma), f \longmapsto f$ is a functor.

(2) $\tau: C T S \longrightarrow$ AChy, $(X, \bar{D}) \longmapsto(X, \tau \bar{D}), f \longmapsto f$ is a functor.

(3) $\sigma \circ \tau=i d_{C T S}$ and $\tau \circ \sigma=i d_{A C h y}$.

(4) $\sigma(u A C h y)=u C T S$ and $\tau(u C T S)=u A C h y$.

6.4. Corollary. The categories AChy and CTS are isomorphic and the categories uAChy and $u C T S$ are isomorphic.

We can also define isomorphism functors between the categories $P C h y^{*}$ and $C T S_{S(0)}$ provided that the t-norm $*$ is continuous Archimedean with additive generator $S$ : $[0,1] \longrightarrow[0, \infty]$. For $(X, \bar{C}) \in\left|P C h y^{*}\right|$ we define $\Gamma_{S} \bar{C}$ by

$$
\mathbb{F} \in\left(\Gamma_{S} \bar{C}\right)_{\epsilon} \quad \Longleftrightarrow \quad \mathbb{F} \in C_{S^{(-1)}(\epsilon)},
$$

and for $(X, \bar{D}) \in\left|C T S_{S(0)}\right|$ we define $\Delta_{S} \bar{D}$ by

$$
\mathbb{F} \in\left(\Delta_{S} \bar{D}\right)_{\alpha} \quad \Longleftrightarrow \quad \mathbb{F} \in D_{S(\alpha)}
$$

The following result is then not difficult to prove.

6.5. Lemma. (1) $\Gamma_{S}: P C h y^{*} \longrightarrow C T S_{S(0)},(X, \bar{C}) \longmapsto\left(X, \Gamma_{S} \bar{C}\right), f \longmapsto f$ is a functor.

(2) $\Delta_{S}: C T S_{S(0)} \longrightarrow P C h y^{*},(X, \bar{D}) \longmapsto\left(X, \Delta_{S} \bar{D}\right), f \longmapsto f$ is a functor.

(3) $\Gamma_{S} \circ \Delta_{S}=i d_{C T S_{S(0)}}$ and $\Delta_{S} \circ \Gamma_{S}=i d_{P C h y^{*}}$.

(4) $\Gamma_{S}\left(P C h y^{\wedge}\right)=u C T S$ and $\Delta_{S}(u C T S)=P C h y^{\wedge}$.

Noting that $C T S_{\infty}=C T S$ we can state the following results.

6.6. Corollary. For a strict t-norm *, the categories PChy* and CTS are isomorphic. For a nilpotent $t$-norm $*$, the categories PChy* and $C T S_{S(0)}$ are isomorphic. Furthermore, the categories $P C h y^{\wedge}$ and $u C T S$ are isomorphic.

6.7. Theorem. For strict $t$-norms, all categories PChy* are isomorphic. For nilpotent t-norms all categories PChy* are isomorphic. 


\section{Probabilistic uniform limit spaces, uniform limit tower spaces and approach uniform limit spaces}

A probabilistic uniform limit space under the t-norm * [14] is a pair $(X, \bar{L})$ of a set $X$ and a non-void family of subsets of $\mathbb{F}(X \times X), \bar{L}=\left(L_{\alpha}\right)_{\alpha \in[0,1]}$ that satisfies the following axioms.

(PUL1) $\quad[x] \times[x] \in L_{\alpha}$ for all $x \in X$ and all $\alpha \in[0,1]$;

(PUL2) $\mathbb{G} \in L_{\alpha}$ whenever $\mathbb{F} \leq \mathbb{G}$ and $\mathbb{F} \in L_{\alpha}$;

(PUL3) $L_{\alpha} \subseteq L_{\beta}$ whenever $\beta \leq \alpha$;

(PUL4) $\quad L_{0}=\mathbb{F}(X \times X)$;

(PUL5) $\mathbb{F} \wedge \mathbb{G} \in L_{\alpha}$ whenever $\mathbb{F}, \mathbb{G} \in L_{\alpha}$

(PUL6) $\mathbb{F}^{-1} \in L_{\alpha}$ whenever $\mathbb{F} \in L_{\alpha}$

(PUL7) $\mathbb{F} \circ \mathbb{G} \in L_{\alpha * \beta}$ whenever $\mathbb{F} \in L_{\alpha}, \mathbb{G} \in L_{\beta}$ and $\mathbb{F} \circ \mathbb{G}$ exists;

(PULLC) $L_{\alpha}=\bigcap_{\beta<\alpha} L_{\beta}$.

A mapping $f: X \longrightarrow X^{\prime}$ between two probabilistic uniform limit spaces $(X, \bar{L})$ and $\left(X^{\prime}, \bar{L}^{\prime}\right)$ is called uniformly continuous if $(f \times f)\left(L_{\alpha}\right) \subseteq L_{\alpha}^{\prime}$ for all $\alpha \in[0,1]$. The category of all probabilistic uniform limit spaces under the t-norm $*$ with uniformly continuous mappings as morphisms is denoted by $P U L I M^{*}$.

7.1. Lemma. Let $(X, \bar{L})$ be a probabilistic uniform limit space under the t-norm $\wedge$. Then (PUL7) is equivalent to the axiom

$\left(\right.$ uPUL7) $\quad \mathbb{F} \circ \mathbb{G} \in L_{\alpha}$ whenever $\mathbb{F} \in L_{\alpha}$ and $\mathbb{G} \in L_{\alpha}$ and $\mathbb{F} \circ \mathbb{G}$ exists.

Proof. Similar to the proof of Lemma 4.1.

Therefore, probabilistic uniform limit spaces under the t-norm $\wedge$ are (left-continuous) componentwise probabilistic uniform limit spaces in the definition of [14]. The category $P U L I M^{*}$ is topological and not hereditary and products of quotients are quotients. $P U L I M^{\wedge}$ is Cartesian closed [14].

A uniform limit tower space [7] is a pair $(X, \bar{M})$ of a set $X$ and a non-void family of subsets of $\mathbb{F}(X \times X), \bar{M}=\left(M_{\epsilon}\right)_{\epsilon \in[0, \infty]}$ that satisfies the following axioms.

(ULT1) $[x] \times[x] \in M_{\epsilon}$ for all $x \in X$ and all $\epsilon \in[0, \infty]$;

(ULT2) $\mathbb{G} \in M_{\epsilon}$ whenever $\mathbb{F} \leq \mathbb{G}$ and $\mathbb{F} \in M_{\epsilon}$;

(ULT3) $M_{\epsilon} \subseteq M_{\delta}$ whenever $\epsilon \leq \delta$;

(ULT4) $M_{\infty}=\mathbb{F}(X \times X)$;

(ULT5) $\mathbb{F} \wedge \mathbb{G} \in \epsilon$ whenever $\mathbb{F}, \mathbb{G} \in M_{\epsilon}$;

(ULT6) $\mathbb{F}^{-1} \in M_{\epsilon}$ whenever $\mathbb{F} \in M_{\epsilon}$;

(ULT7) $\mathbb{F} \circ \mathbb{G} \in M_{\epsilon+\delta}$ whenever $\mathbb{F} \in M_{\epsilon}, \mathbb{G} \in M_{\delta}$ and $\mathbb{F} \circ \mathbb{G}$ exists;

(ULTLC) $M_{\epsilon}=\bigcap_{\epsilon<\delta} M_{\delta}$. 
A mapping $f: X \longrightarrow X^{\prime}$ between two uniform limit tower spaces $(X, \bar{M})$ and $\left(X^{\prime}, \bar{M}^{\prime}\right)$ is called uniformly continuous if $(f \times f)\left(M_{\epsilon}\right) \subseteq M_{\epsilon}^{\prime}$ for all $\epsilon \in[0, \infty]$. The category of all uniform limit tower spaces with uniformly continuous mappings as morphisms is denoted by $U L T S$.

If we replace the axiom (ULT6) by the axiom

(uULT6) $\mathbb{F} \circ \mathbb{G} \in M_{\epsilon \vee \delta}$ whenever $\mathbb{F} \in M_{\epsilon}, \mathbb{G} \in M_{\delta}$ and $\mathbb{F} \circ \mathbb{G}$ exists;

then we speak of a ultra-uniform limit tower space. The category of ultra-uniform limit tower spaces with uniformly continuous mappings as morphisms is denoted by $u U L T S$.

7.2. Lemma. Let $(X, \bar{M})$ be an ultra-uniform limit tower space. Then (uULT6) is equivalent to the axiom

$\left(u U L T 6^{\prime}\right) \quad \mathbb{F} \circ \mathbb{G} \in M_{\epsilon}$ whenever $\mathbb{F} \in M_{\epsilon}$ and $\mathbb{G} \in M_{\epsilon}$ and $\mathbb{F} \circ \mathbb{G}$ exists.

Proof. Similar to the proof of Lemma 7.1.

We again define, for $0<\omega \leq \infty$, an $\omega$-uniform limit tower space $(X, \bar{M})$ as a uniform limit tower space that satisfies the following strengthening of the axiom (ULT4):

(ULT4 $\omega) \quad M_{\epsilon}=\mathbb{F}(X \times X)$ whenever $\omega \leq \epsilon$.

The subcategory of $U L T S$ with objects the $\omega$-uniform limit tower spaces is denoted by $U L T S_{\omega}$.

An approach uniform limit space [7] is a pair $(X, \eta)$ of a set $X$ and a mapping $\eta$ : $\mathbb{F}(X \times X) \longrightarrow[0, \infty]$ that satisfies the following axioms.

(AULS1) $\eta([x] \times[x])=0$ for all $x \in X$;

(AULS2) $\quad \eta(\mathbb{G}) \leq \eta(\mathbb{F})$ whenever $\mathbb{F} \leq \mathbb{G} ;$

(AULS3) $\quad \eta(\mathbb{F} \wedge \mathbb{G}) \leq \eta(\mathbb{F}) \vee \eta(\mathbb{G})$;

(AULS4) $\eta\left(\mathbb{F}^{-1}\right)=\eta(\mathbb{F})$;

(AULS5) $\quad \eta(\mathbb{F} \circ \mathbb{G}) \leq \eta(\mathbb{F})+\eta(\mathbb{G})$ whenever $\mathbb{F} \circ \mathbb{G}$ exists.

A mapping $f: X \longrightarrow X^{\prime}$ between two approach uniform limit spaces $(X, \eta),\left(X^{\prime}, \eta^{\prime}\right)$ is called a uniform contraction if for all $\mathbb{F} \in \mathbb{F}(X \times)$ we have $\eta^{\prime}((f \times f)(\mathbb{F})) \leq \eta(\mathbb{F})$

If we replace the axiom (AULS5) by the stronger axiom $($ uAULS5) $\quad \eta(\mathbb{F} \circ \mathbb{G}) \leq \eta(\mathbb{F}) \vee \eta(\mathbb{G})$ whenever $\mathbb{F} \circ \mathbb{G}$ exists; then we call the pair $(X, \eta)$ an ultra-approach uniform limit space.

The category of approach uniform limit spaces with uniform contractions as morphisms is denoted by $A U L S$, the subcategory of ultra-approach convergence spaces is denoted by $u A U L S$. It is shown in [7] that $u A U L S$ is a bireflective subcategory of $A U L S$ and that it is a topological construct and is Cartesian closed. It is mentioned that $A U L S$ is a topological construct. 
We can again define isomorphism functors between these categories. Lee and Windels [7] mention the following. For $(X, \eta) \in|A U L S|$ we define the uniform limit tower $\kappa \eta$ by

$$
\mathbb{F} \in(\kappa \eta)_{\epsilon} \quad \Longleftrightarrow \quad \eta(\mathbb{F}) \leq \epsilon .
$$

For $(X, \bar{M}) \in|U L T S|$ we define the approach uniform limit $\chi \bar{M}: \mathbb{F}(X \times X) \longrightarrow[0, \infty]$ by

$$
\chi \bar{M}(\mathbb{F})=\bigwedge\left\{\epsilon \in[0, \infty]: \mathbb{F} \in M_{\epsilon}\right\}
$$

This again gives rise to two isomorphism functors, $\kappa: A U L S \longrightarrow U L T S$ and $\chi:$ $U L T S \longrightarrow A U L S$ and we obtain the following result.

7.3. Lemma. (1) $\quad \kappa: A U L S \longrightarrow U L T S,(X, \eta) \longmapsto(X, \kappa \eta), f \longmapsto f$ is a functor.

(2) $\chi: U L T S \longrightarrow A U L S,(X, \bar{M}) \longmapsto(X, \chi \bar{M}), f \longmapsto f$ is a functor.

(3) $\kappa \circ \chi=i d_{U L T S}$ and $\chi \circ \kappa=i d_{A U L S}$.

(4) $\kappa(u A U L S)=u U L T S$ and $\chi(u U L T S)=u A U L S$.

We obtain as a corollary the following theorem.

7.4. Theorem. The categories AULS and ULTS are isomorphic and the categories $u A U L S$ and $u U L T S$ are isomorphic.

Now, once again let the continuous Archimedean t-norm $*$ have the additive generator $S$. For $(X, \bar{L}) \in\left|P U L I M^{*}\right|$ we define the $S(0)$-uniform limit tower $\Omega_{S} \bar{L}$ by

$$
\mathbb{F} \in\left(\Omega_{S} \bar{L}\right)_{\epsilon} \quad \Longleftrightarrow \quad \mathbb{F} \in L_{S(-1)}(\epsilon)
$$

and for $(X \bar{M}) \in\left|U L T S_{S(0)}\right|$ we define the probabilistic uniform limit structure $\Lambda_{S} \bar{M}$ by

$$
\mathbb{F} \in\left(\Lambda_{S} \bar{M}\right)_{\alpha} \Longleftrightarrow \mathbb{F} \in M_{S(\alpha)} \text {. }
$$

This gives rise to two isomorphism functors and we can prove the following result.

7.5. Lemma. (1) $\Omega_{S}: P U L I M^{*} \longrightarrow U L T S_{S(0)},(X, \bar{L}) \longmapsto\left(X, \Omega_{S} \bar{L}\right), f \longmapsto f$ is a functor.

(2) $\Lambda_{S}: U L T S_{S(0)} \longrightarrow P U L I M^{*},(X, \bar{M}) \longmapsto\left(X, \Lambda_{S} \bar{M}\right), f \longmapsto f$ is a functor.

(3) $\Omega_{S} \circ \Lambda_{S}=i d_{U L T S_{S(0)}}$ and $\Lambda_{S} \circ \Omega_{S}=i d_{P U L I M^{*}}$.

(4) $\Omega_{S}\left(P U L I M^{\wedge}\right)=u U L T S$ and $\Lambda_{S}(u U L T S)=P U L I M^{\wedge}$.

Noting again that $U L T S_{\infty}=U L T S$ we obtain the following results.

7.6. Corollary. For a strict t-norm *, the categories PULIM* and ULTS are isomorphic. For a nilpotent t-norm *, the categories PULIM* and ULTS $S_{S(0)}$ are isomorphic. Furthermore, the categories $P U L I M^{\wedge}$ and $u U L T S$ are isomorphic. 
7.7. Theorem. For strict $t$-norms, all categories PULIM* are isomorphic. For nilpotent $t$-norms all categories $P U L I M^{*}$ are isomorphic.

\section{Conclusions}

We showed in this paper, that for certain classes of t-norms, all categories of probabilistic limit spaces under these t-norms are isomorphic. We could show this for the class of strict t-norms and for the class of nilpotent t-norms. This essentially means that it is sufficient to study "prototype spaces", i.e. it would be sufficient to study probabilistic limit spaces under the product t-norm (as a prototype for probabilistic limit spaces under strict t-norms) or probabilistic limit spaces under the Lukasiewics t-norm (as a prototype for probabilistic limit spaces under nilpotent t-norms). The proofs depend on the existence of an additive generator. It would be interesting to know if there are other classes of t-norms for which the categories of probabilistic limit spaces are isomorphic. It shall be further remarked that we considered only left-continuous probabilistic limit spaces. This restriction was used in order to accomodate approach limit spaces. The isomorphism functors between the categories of limit tower spaces and of probabilistic

limit spaces, however, also work without imposing the left-continuity condition on the spaces.

\section{References}

[1] Adámek.,J., Herrlich, H. and Strecker, G.E. Abstract and Concrete Categories, Wiley (New York 1989).

[2] Brock, P. and Kent, D.C. Approach spaces, limit tower spaces, and probabilistic convergence spaces, Appl. Cat. Structures 5, 99 - 110, 1997.

[3] Florescu, L.C. Probabilistic convergence structures, Aequ. Math. 38, 123 - 145, 1989.

[4] Frank, M.J. Probabilistic topological spaces, J. Math. Anal. Appl. 34, 67 - 81, 1971.

[5] Kent D.C. and Richardson, G.D. Completion of probabilistic Cauchy spaces, Math. Japonica 48, 399-407, 1998.

[6] Klement, E.P., Mesiar R. and Pap, E. Triangular Norms, Springer (2000).

[7] Lee, Y.J. and Windels, B. Transitivity in approach theory, Int. J. Math. \& Math. Sci. 32, $707-720,2002$.

[8] Lowen E. and Lowen, R. A quasitopos containing CONV and MET as full subcategories, Int. J. Math. \& Math. Sci. 11, 417 - 438, 1988.

[9] Lowen, R. Approach spaces. A common supercategory of TOP and MET, Math. Nachr. 141, $183-226,1989$. 
[10] Lowen, R. Approach Spaces. The Missing Link in the Topology-Uniformity-Metric Triad, Claredon Press (Oxford 1997).

[11] Lowen R. and Lee, Y.J. Approach theory in merotopic, Cauchy and convergence spaces. II, Acta Math. Hungar. 83, 209 - 229, 1999.

[12] Menger, K. Statistical metrics, Proc. Nat. Acad. Sci. USA 37, 178 - 180, 1951.

[13] Minkler, J., Minkler G. and Richardson, G. Subcategories of filter tower spaces, Appl. Cat. Structures 9, 369 - 379, 2001.

[14] Nusser, H. A generalization of probabilistic uniform spaces, Appl. Cat. Structures 10, 81 - 98, 2002.

[15] Richardson, G.D. and Kent, D.C. Probabilistic convergence spaces, J. Austral. Math. Soc. 61, $400-420,1996$.

[16] Schweizer, B. and Sklar, A. Probabilistic metric spaces, North Holland (New York 1983). 